\title{
Importancia de la lignina en las contracciones de la madera: revisión bibliográfica
}

\author{
Guadalupe Bárcenas Pazos ${ }^{1}$ \\ Raymundo Dávalos Sotelo ${ }^{1}$
}

\begin{abstract}
RESUMEN
Con la información presentada en este trabajo, producto de una revisión bibliográfica, se demuestra que parte de las contracciones de la madera se puede explicar en función del contenido de lignina. La madera de angiospermas templadas, tanto de México como de Estados Unidos sufre contracciones mayores que la de angiospermas tropicales y que la de coníferas de ambos países. La rigidez tridimensional de la lignina, superior a la de los otros componentes químicos de la pared celular, conjuntamente con su escasa higroscopicidad, restringe los movimientos debidos a los cambios de humedad. Se hicieron análisis de regresión para determinar el grado de influencia de la densidad y del contenido de lignina en las contracciones radial y tangencial y se concluyó que ambas tienen importancia. La densidad resulta ser un factor de mayor importancia, pero la influencia de la lignina no es despreciable. Se destaca que es necesario efectuar estudios experimentales que determinen con toda precisión el efecto de estos factores sobre los cambios dimensionales, conjuntamente con los otros que parecen tener importancia: los extractivos y el volumen de radios.
\end{abstract}

PALABRAS CLAVE:

Contracciones, contenido de lignina, madera de angiospermas templadas, madera de angiospermas tropicales, madera de coníferas, México, Estados Unidos

\section{ABSTRACT}

With the information presented in this paper, obtained from the technical literature, it is proved that shrinkage of wood can be explained partially from the lignin content. Temperate hardwood species, both from México and the United States undergo a greater shrinkage percentage than tropical hardwoods and softwoods from both countries. The three-dimensional stiffness of lignin, greater than that of the other chemical constituents of the cell wall, along with its low higroscopicity, restricts the movements due to changes in moisture content. Regression analyses to determine the influence of specific gravity and lignin content were performed, concluding that both have a marked importance. Specific gravity shows to be the most important variable, although the influence of lignin is also significant. It is remarked that it is necessary to carry out experimental studies on the effect of these variables on dimensional changes, along with that of other important variables, such as extractives and ray volume.

KEY WORDS:

Shrinkage percentage, lignin content, temperate hardwoods, tropical hardwoods, softwoods, México, USA 


\section{INTRODUCCION}

La madera durante su vida útil sufre cambios dimensionales, aumento 0 disminución, como respuesta a las variaciones de contenido de humedad $(\mathrm{CH})$ que se registran en su interior. Estos cambios dimensionales se originan debido a que la madera está formada por polímeros que contienen grupos hidroxilos y otros grupos funcionales con oxígeno que atraen a la humedad a través de puentes de hidrógeno. Los cambios dimensionales de la madera se realizan cuando las paredes celulares de la madera empiezan a perder el agua presente en ellas. A este punto se le denomina punto de saturación de la fibra (PSF).

No todas las especies de madera se contraen en la misma magnitud ante cambios semejantes de contenido de humedad. La proporción en que se contrae la madera depende de factores como: la densidad básica (relativa) de las diferentes especies, orientación de las microfibrillas en la pared celular, el contenido de extractivos, diferencias entre duramen y albura, presencia y dimensiones de los anillos de crecimiento, porcentaje de madera temprana y tardía, presencia de madera juvenil, velocidad de crecimiento de los árboles, posición transversal de la que fueron extraídas las piezas de madera, longitud de las traqueidas, contenido de lignina, cantidad y grado de cristalinidad de la celulosa y porcentaje de radios presente (Panshin y de Zeeuw, 1980; Wiedenbeck et al., 1990).

Para explicar las diferencias de comportamiento en las contracciones en las direcciones radial y tangencial del plano transversal de la madera se han desarrollado varías teorías, como lo resume Boyd (1974), basándose en la estructura celular de la madera. La primera de ellas involucra el papel que desempeñan los radios al restringir los movimientos dimensionales en la dirección radial. Otro grupo de teorías analiza estas diferencias basándose en los diferentes porcentajes de contracción entre la madera temprana y la madera tardía así como en la diferencia de contenido de lignina presente en las paredes radiales y tangenciales de las células; en este grupo también se analiza la influencia de la orientación de las microfibrillas en los valores de contracción. Un grupo más de teorías se basa en la disposición de las capas de las paredes celulares, dándole importancia a la lámina media, la cual es la capa con mayor contenido de lignina de las capas que forman las paredes celulares de la madera (Panshin y de Zeeuw, 1970; Skaar, 1988).

Las diferencias de valores de contracción entre especies han sido estudiadas reiteradamente, sin embargo, muy poco se ha hecho para analizar las diferencias entre grupos de especies, gimnospermas y angiospermas. Schroeder (1972) comparó los valores de contracciones entre estos dos grupos considerando las maderas más abundantes en los Estados Unidos, encontrando que a rangos semejantes de densidad relativa, las angiospermas se contraen aproximadamente un $20 \%$ más que las gimnospermas. También señala que las primeras contienen menos lignina que las gimnospermas, casi el mismo $20 \%$, adjudicándole a este hecho la explicación principal de los menores valores de contracción que presentan las coníferas en comparación con las angiospermas con una densidad básica (relativa), equivalente.

En la República Mexicana, dada su peculiar situación geográfica y variedad climática, se encuentran presentes tres grupos principales de especies maderables: coníferas, angiospermas templadas y angiospermas tropicales. Los dos primeros grupos con características semejantes a algunas de las especies maderables que crecen en los Estados Unidos. Hasta la fecha se ha generado una buena cantidad de información técnica sobre el comportamiento dimensional, composición celular y química de algunas de estas 
maderas. En general, se considera que conociendo adecuadamente el comportamiento y estructura celular de cada uno de los grupos de especies, se puede realizar un análisis de la influencia que los componentes químicos de la pared celular tienen sobre las contracciones en las maderas mexicanas.

\section{OBJETIVO}

Con base en la información bibliográfica existente sobre los valores de contracción y composición química de algunas maderas mexicanas se intenta determinar el efecto del contenido de lignina sobre los valores de la contracción transversal de la madera.

\section{ANTECEDENTES}

Se han realizado un buen número de trabajos para analizar los cambios dimensionales que presenta la madera como respuesta a los cambios de humedad en su interior, que a su vez son causados por los cambios de humedad en el ambiente circundante, cuando el $\mathrm{CH}$ se encuentra por abajo del PSF, así como para analizar y definir las causas de las diferencias de comportamiento tanto entre especies como dentro de una misma pieza de madera. En 1949, Dickinson et al., determinaron que las angiospermas tropicales presentaban valores de contracción más pequeños que aquellos registrados para angiospermas templadas de los Estados Unidos. Los autores sólo mencionaron este hecho sin profundizar en las posibles causas.

Higgins (1957) resaltó el hecho de que las angiospermas tropicales, en general, tienen una alta razón de lignina a celulosa, lo que se considera que implica un número reducido de iones hidróxilo. Por su parte Wiedenbeck et al., 1990, realizaron un estudio sobre algunos factores geográficos que afectan las contracciones de la madera de Pinus contorta: latitud, altitud, diámetro del árbol, encontrando que para una variedad, estas variables no tienen ningún efecto, pero en otra, la latitud y el tamaño del árbol, tienen cierta influencia en los valores de contracción. En el mismo estudio, estos investigadores determinaron que la densidad básica o relativa es el factor más importante que afecta las contracciones radiales y volumétricas así como la relación entre la contracción tangencial y la radial. En ambos casos la correlación es positiva.

Fergus et al. en 1969, analizaron los componentes químicos de la madera de una conífera (Picea mariana Mill.), determinando que más del $70 \%$ de la lignina se encontraba en la pared secundaria de sus traqueidas de madera temprana con niveles de más del $80 \%$ en las células de madera tardía que eran a su vez más densas, más resistentes y más rígidas que las células de madera temprana. Fergus y Goring (1970) encontraron que del total de lignina presente en la madera de una angiosperma (Betula papyrifera Marsh.), el 59.9\% estaba contenido en las paredes celulares y el resto se encontraba distribuida en las células de los radios, la pared secundaria y la lámina media de los vasos y en la lámina media de las fibras.

Nearn en 1955, encontró que los valores de contracción volumétrica presentan una relación negativa con el contenido de lignina. Kelsey (1963) también reporta una relación inversa entre las contracciones volumétricas y el contenido de lignina; este argumento fue confirmado posteriormente por Bland (1971) y analizado más tarde por Chafé en 1987.

Bosshard (1956) menciona que las paredes radiales de las células de la madera presentan un contenido de lignina mayor que el contenido en las paredes tangenciales lo que explica los valores menores de contracción en la dirección radial ya que la lignina al ser más rígida y menos higroscópica que las holocelulosas, limita las contracciones. Kato y Nakato 
(1968) confirman esta aseveración realizando un estudio con coníferas, encontrando que efectivamente las paredes radiales de las células se encuentran más lignificadas que las tangenciales.

Boyd (1974) contribuye con mayor información respaldando esta teoría, afirmando que los precursores de la lignina se encuentran entre las microfibrillas en las paredes radiales durante el crecimiento de las células y una vez que se polimerizan, se transforman en un agente "rellenador" proporcionando mayor rigidez a las paredes radiales y disminuyendo su higroscopicidad. Este autor concluyó que los efectos cuantitativos asociados con la contracción anisotrópica están dominados, en primer lugar, por el grado de lignificación de las paredes tangenciales y radiales.

Quirk (1984), por su parte, encontró que la lignina contenida en la lámina media contribuye significativamente en la diferencia de valores de contracción transversal entre células de madera temprana y madera tardía, presentando datos que indican que la lignina contribuye a que las paredes de las traqueidas de madera temprana de Pseudotsuga menziesii (Douglas-Fir) tengan valores mayores de contracción que las células de madera tardía, haciendo estas mediciones por medios optométricos. Quirk explica que la expansión de los lúmenes de la las células de madera temprana y el comportamiento del perímetro celular al secarse indican que los cambios dimensionales de las paredes de las células de la madera temprana son restringidos por la lámina media con mayor contenido de lignina . Consecuentemente, las paredes de madera temprana se contraen más con la pérdida de humedad que las de madera tardía.

De estos antecedentes, se puede concluir que el porcentaje de contenido de lignina presente en las paredes celulares o en la lámina media, contribuye de manera significativa en el comportamiento dimensional de la madera.

\section{REVISION BIBLIOGRAFICA}

Se revisó la información disponible de aquellas especies mexicanas sobre las que hubiera datos publicados, tanto de sus valores de contracción transversal, radial y tangencial como de su composición química, principalmente el contenido de lignina. Se encontró información sobre 5 coníferas, 15 angiospermas tropicales y 10 angiospermas templadas provenientes de diferentes localidades geográficas del país (Acosta, 1964; Novelo, 1964; Echenique y Díaz, 1969; Echenique, 1970; Porres y Valladares, 1979; Bárcenas et al., 1980; Candelario, 1980; Delgado, 1980; Fuentes, 1980; Villalvazo y Faix, 1981; SARH, 1982; Pettersen, 1984; Castañeda, 1986; Ordóñez et al. 1990; Fuentes, 1990; Montes, 1991; Islas, 1992; Bárcenas s/f; Bárcenas, 1995; de la Paz et al., 1995; Hernández, 1995; Vidrio, 1995; de la Paz et al., 1999). En los casos para los que se contaba con más de un valor de contracciones y de contenido de lignina, se determinó el valor promedio. Con la información de las contracciones, se calculó el valor del PSF para cada una de las maderas, utilizando el método gráfico de la intersección de ejes coordenados, tomando como base los valores de contracción intermedia y total.

Con el fin de comparar el comportamiento de las coníferas y las angiospermas templadas que crecen en México con las especies semejantes que crecen en los Estados Unidos, se recopiló la información sobre estas especies (Panshin y de Zeeuw, 1970; Pettersen, 1984; FPL, 1989) y se agruparon conforme a los criterios de densidad básica definidos en Dávalos (1994). Los detalles del número de especies norteamericanas y su forma de agrupación de describen en Bárcenas y Dávalos (1999a; 1999b). 
Se hicieron análisis de regresión tomado como variables independientes la densidad básica y el contenido de lignina y como variables dependientes las contracciones radial y tangencial. Se calcularon los promedios de los datos obtenidos por grupos de especies y por rangos de densidades y con ellos se construyeron gráficas para comparar el comportamiento de los grupos de especies.

\section{RESULTADOS}

En la tabla 1 se presentan los valores obtenidos de la literatura, así como los promedios calculados para maderas que crecen en México. En la tabla 2 se presentan los valores promedio por grupos de densidad relativa para las especies de EUA. En la figura 1 se presentan gráficamente los valores promedio de contracciones y contenido de lignina obtenidos de los cinco grupos de maderas, separados cada uno de ellos por rangos de densidad básica o relativa (DR: pa/vv).

Se hicieron análisis de regresión entre las variables de interés para este estudio: las variables independientes son la densidad básica y el contenido de lignina y las dependientes son los valores de contracción radial y tangencial. En la tabla 3 se presenta los resultados del análisis de regresión múltiple entre la contracción tangencial y las dos variables independientes. En la tabla 4 se incluye el resultado del análisis de regresión múltiple para la contracción radial. En las figuras 2 y 3 se presentan las gráficas de dispersión entre densidad y contracción tangencial y contenido de lignina y contracción tangencial, respectivamente. En ambas gráficas se han incluido las líneas de tendencia con la regresión lineal simple entre las dos variables y su correspondiente coeficiente de determinación $\left(R^{2}\right)$.

\section{DISCUSION}

Existen dos categorías muy bien definidas, en cuanto a contenido de lignina: la primera incluye a las angiospermas de clima templado y la segunda consiste de las angiospermas tropicales y las coníferas. Ambas tienen comportamientos bastante diferentes por lo que respecta a las contracciones: el grupo de las angiospermas templadas, el cual contiene especies de encino en su mayoría, tiene contracciones más altas en promedio que las de las especies incluidas en los otros grupos. Las primeras presentan contenidos de lignina menores y por lo tanto, según la tesis central de este artículo son más susceptibles de contraerse o expandirse, al no tener tan importante acción restrictiva de la lignina, la cuál se caracteriza por su rigidez. La observación de Higgins (1957) de que las maderas tropicales contienen más lignina, significa que hay menos enlaces disponibles para atraer la humedad, lo que contribuye a explicar porque estas especies tienen una muy buena estabilidad dimensional, es decir, su relación entre los valores de contracción tangencial y radial es menor o igual que 2.

Los valores de contracciones tanto radiales como tangenciales y por consiguiente, volumétricas, son muy parecidos entre sí para los tres grupos de especies incluidos entre los de menor contracción y mayor contenido de lignina: coníferas mexicanas, coníferas norteamericanas y angiospermas tropicales. Esto es sorprendente para especies con características anatómicas tan diferentes. Los indicadores de estabilidad dimensional (coeficiente $T / R$ ) son muy similares, resultando las coníferas norteamericanas el grupo más estable, incluso, ligeramente más estables que las maderas tropicales mexicanas, las cuales tienen un mayor grado de variabilidad. 
Tabla 1. Valores de contracciones y contenido de lignina para especies maderables mexicanas agrupadas por grupos de especies y rangos de Densidad relativa o básica

\begin{tabular}{|c|c|c|c|c|c|c|}
\hline \multicolumn{7}{|c|}{ "ANGIOSPERMAS TEMPLADAS } \\
\hline ESPECIES & $\begin{array}{c}\text { DR } \\
(\mathrm{pa} / \mathrm{vv})\end{array}$ & $\begin{array}{c}\text { PSF est. } \\
\%\end{array}$ & $\begin{array}{c}\text { C. TANG. } \\
\%\end{array}$ & $\begin{array}{l}\text { C. RAD. } \\
\%\end{array}$ & $\begin{array}{l}\text { REL. } \\
\mathrm{CT} / \mathrm{CR}\end{array}$ & $\begin{array}{c}\text { C. LIGN. } \\
\%\end{array}$ \\
\hline \multicolumn{7}{|l|}{$>0.700$} \\
\hline Quercus resinosa & 0.762 & 39 & 14.3 & 6.3 & 2.4 & $22^{(11,3,4)}$ \\
\hline Q. obtusata & 0.756 & 33 & 15.3 & 6.7 & 2.3 & $22^{(11,3)}$ \\
\hline Promedio & 0.759 & 36 & 14.8 & 6.5 & 2.4 & 22 \\
\hline \multicolumn{7}{|l|}{$0.450-0.699$} \\
\hline$Q$ crassifolia & 0.648 & 30 & 12.4 & 5.7 & 2.2 & $21^{(5)}$ \\
\hline Q. glabrescens & 0.647 & 25 & 11.3 & 5.1 & 2.2 & $20^{(5)}$ \\
\hline Q. candicans & 0.639 & 32 & 12.8 & 5.1 & 2.5 & $25^{(11,5)}$ \\
\hline Q. laurina & 0.627 & 35 & 14.0 & 4.9 & 2.9 & $18^{(11,5)}$ \\
\hline Q. mexicana & 0.617 & 36 & 15.3 & 5.4 & 2.9 & $22^{(5)}$ \\
\hline Q. affinis & 0.616 & 31 & 14.0 & 5.6 & 2.5 & $20^{(5)}$ \\
\hline Liquidambar styraciflua & 0.469 & 37 & 110.0 & 5.4 & 1.8 & $24^{(1)}$ \\
\hline Promedio & 0.609 & 32 & 13.0 & 5.3 & 2.5 & 21 \\
\hline \multicolumn{7}{|l|}{$<0.450$} \\
\hline Alnus jorullensis & 0.400 & 31 & 8.2 & 5.9 & 1.4 & $32^{(1)}$ \\
\hline \multicolumn{6}{|c|}{ Promedio contenido de lignina } & 23 \\
\hline \multicolumn{7}{|c|}{ ANGIOSPERMAS TROPICALES } \\
\hline \multicolumn{7}{|l|}{$>0.700$} \\
\hline Manilkara zapota & 0.903 & 24 & 9.4 & 5.8 & 1.6 & $27^{(9)}$ \\
\hline Ampelocera hottlei & 0.728 & 20 & 89 & 5.2 & 1.7 & $31^{(9)}$ \\
\hline Brosimum alicastrum $^{(9)}$ & 0.727 & 20 & 8.0 & 5.1 & 1.6 & $26^{(9,8,2)}$ \\
\hline Hymenea courbaril & 0.700 & 25 & 8.3 & 4.4 & 1.9 & $20^{(8)}$ \\
\hline Promedio & 0.766 & 22 & 8.7 & 5.2 & 1.7 & 26 \\
\hline \multicolumn{7}{|l|}{$0.450-0.699$} \\
\hline Vitex gaumeri & 0.660 & 21 & 8.5 & 4.1 & 2.1 & $31^{(1)}$ \\
\hline Terminalia amazonia & 0.630 & 22 & 7.4 & 4.7 & 1.6 & $25^{(8,9)}$ \\
\hline Cordia alliodora & 0.553 & 21 & 8.2 & 3.9 & 2.1 & $30^{(8)}$ \\
\hline Lysiloma acapulcensis & 0.517 & 26 & 5.5 & 3.7 & 1.5 & $20^{(1)}$ \\
\hline Vochysia hondurensis & 0.515 & 32 & 10.2 & 4.0 & 2.6 & $22^{(8)}$ \\
\hline Poulsenia armata & 0.445 & 26 & 8.5 & 4.0 & 2.1 & $36^{(8)}$ \\
\hline Promedio & 0.569 & 25 & 8.0 & 4.1 & 2.0 & 27 \\
\hline
\end{tabular}


... cont. Tabla 1. Valores de contracciones y contenido de lignina para especies maderables mexicanas agrupadas por grupos de especies y rangos de Densidad relativa o básica

\begin{tabular}{|c|c|c|c|c|c|c|}
\hline ESPECIES & $\begin{array}{c}\text { DR } \\
(\mathrm{pa} / \mathrm{vv})\end{array}$ & $\begin{array}{c}\text { PSF } \\
\text { est. } \\
\%\end{array}$ & $\begin{array}{c}\text { C. } \\
\text { TANG. } \\
\%\end{array}$ & $\begin{array}{c}\text { C. RAD. } \\
\%\end{array}$ & $\begin{array}{l}\text { REL. } \\
\text { CT/CR }\end{array}$ & $\begin{array}{c}\text { C. } \\
\text { LIGN. } \\
\%\end{array}$ \\
\hline \multicolumn{7}{|l|}{$<0.450$} \\
\hline Simaruba glauca & 0.400 & 26 & 6.4 & 2.8 & 2.4 & $29^{(1)}$ \\
\hline Cedrela odorata & 0.374 & 26 & 5.0 & 4.0 & 1.3 & $36^{(1)}$ \\
\hline Bursera simarouba & 0.366 & 32 & 5.1 & 2.8 & 1.8 & $23^{(8)}$ \\
\hline Schizolobium parahibum & 0.316 & 23 & 6.9 & 2.9 & 2.6 & $26^{(8)}$ \\
\hline Ceiba pentandra & 0.230 & 32 & 5.2 & 2.1 & 2.5 & $24^{(8)}$ \\
\hline Promedio & 0.337 & 28 & 5.7 & 2.9 & 2.1 & 28 \\
\hline \multicolumn{6}{|l|}{ Promedio contenido de lignina } & 27 \\
\hline \multicolumn{7}{|c|}{ CONIFERAS } \\
\hline \multicolumn{7}{|l|}{$0.450-0.699$} \\
\hline Pinus douglasiana & 450 & 27 & 8.0 & 4.2 & 1.9 & $27^{(5)}$ \\
\hline Pinus patula var. longepedunculata & 0.499 & 25 & 9.1 & 5.5 & 1.7 & $27^{(6)}$ \\
\hline Promedio & 0.475 & 26 & 8.6 & 4.9 & 1.8 & 27 \\
\hline \multicolumn{7}{|l|}{$<0.450$} \\
\hline Pinus leiophylla & 0.440 & 28 & 8.6 & 3.7 & 2.3 & $28^{(7)}$ \\
\hline Pinus rudis & 0.396 & 24 & 7.5 & 3.1 & 2.5 & $26^{(6)}$ \\
\hline Pinus ayacahuite & 0.380 & 22 & 7.6 & 2.9 & 2.7 & $30^{(6)}$ \\
\hline Promedio & 0.404 & 26 & 8.0 & 3.9 & 2.2 & 28 \\
\hline \multicolumn{6}{|l|}{ Promedio contenido de lignina } & 28 \\
\hline
\end{tabular}

(1) Acosta, 1964: (2) Castañeda, 1986; (3) Delgado, 1980; (4) Fuentes, 1980; (5) Hernández, 1995; (6) Islas, 1992; (7) Montes, 1991; ${ }^{(8)}$ Pettersen, 1984; $\left.{ }^{9}\right)$ Porres y Valladares, 1979; ${ }^{(10)}$ Vidrio, 1995; ${ }^{(11)}$ Villalvazo y Faix, 1981 -

Tabla 2. Valores promedio de contracciones y contenido de lignina para especies maderables de Estados Unidos, por grupos de especies y rangos de Densidad relativa o básica

\begin{tabular}{|c|c|c|c|c|c|c|}
\hline \multicolumn{7}{|c|}{ "ANGIOSPERMAS TEMPLADAS } \\
\hline ESPECIES & $\begin{array}{c}\text { DR } \\
(\mathrm{pa} / \mathrm{vv})\end{array}$ & $\begin{array}{c}\text { PSF est. } \\
\%\end{array}$ & $\begin{array}{c}\text { C. TANG. } \\
\%\end{array}$ & $\begin{array}{c}\text { C. RAD. } \\
\%\end{array}$ & $\begin{array}{l}\text { REL. } \\
\text { CT/CR }\end{array}$ & $\begin{array}{l}\text { C. LIGN. } \\
\%\end{array}$ \\
\hline$>0.700$ & 0.800 & 25 & 9.5 & 6.6 & 1.4 & 25 \\
\hline $0.450-0.699$ & 0.550 & 25 & 9.4 & 5.1 & 1.8 & 23 \\
\hline$<0.450$ & 0.380 & 25 & 7.7 & 3.9 & 2.0 & 21 \\
\hline Promedio contenido de lignina & & & & & & 23 \\
\hline \multicolumn{7}{|c|}{ CONIFERAS } \\
\hline $0.450-0.699$ & 0.480 & 25 & 7.5 & 4.5 & 1.7 & 27 \\
\hline$<0.450$ & 0.370 & 25 & 6.6 & 3.5 & 1.9 & 29 \\
\hline Promedio contenido de lignina & & & & & & 28 \\
\hline
\end{tabular}




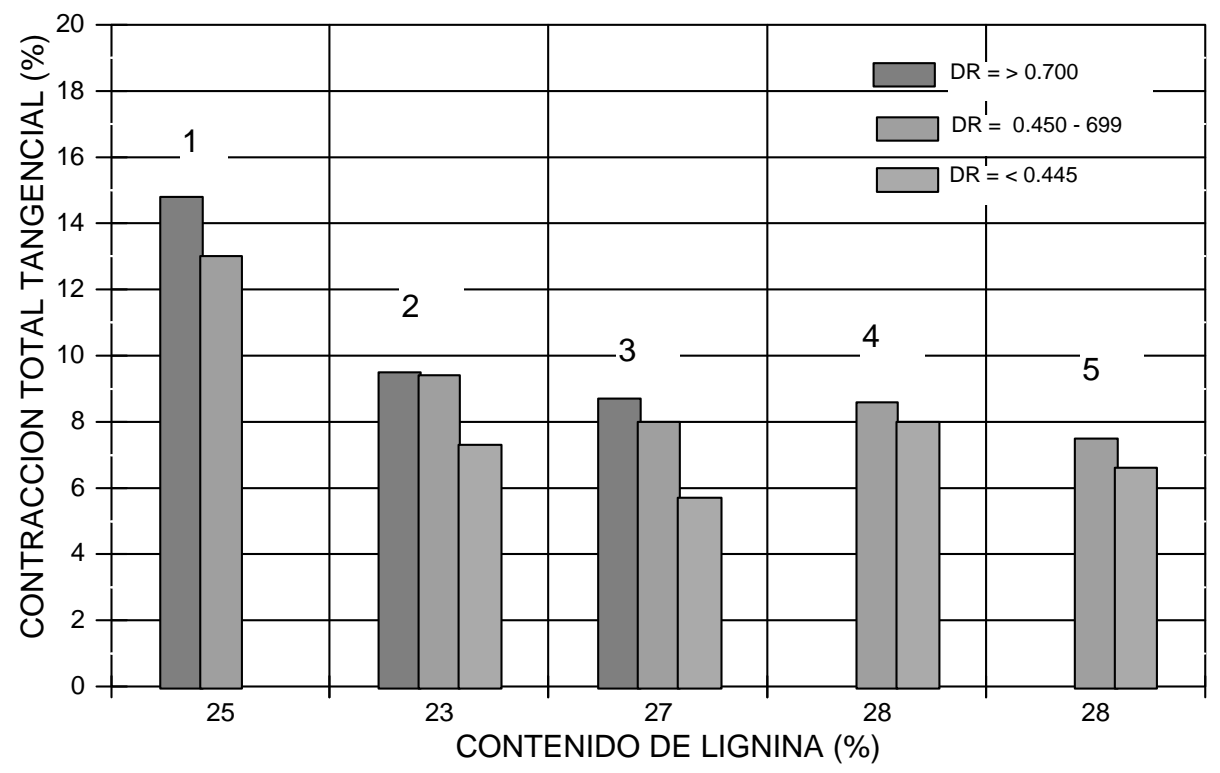

1 ANGIOSPERMAS TEMPLADAS MEXICO ANGIOSPERMAS TEMPLADAS EUA ANGIOSPERMAS TROPICALES MEXICO CONIFERAS MEXICO

CONIFERAS EUA

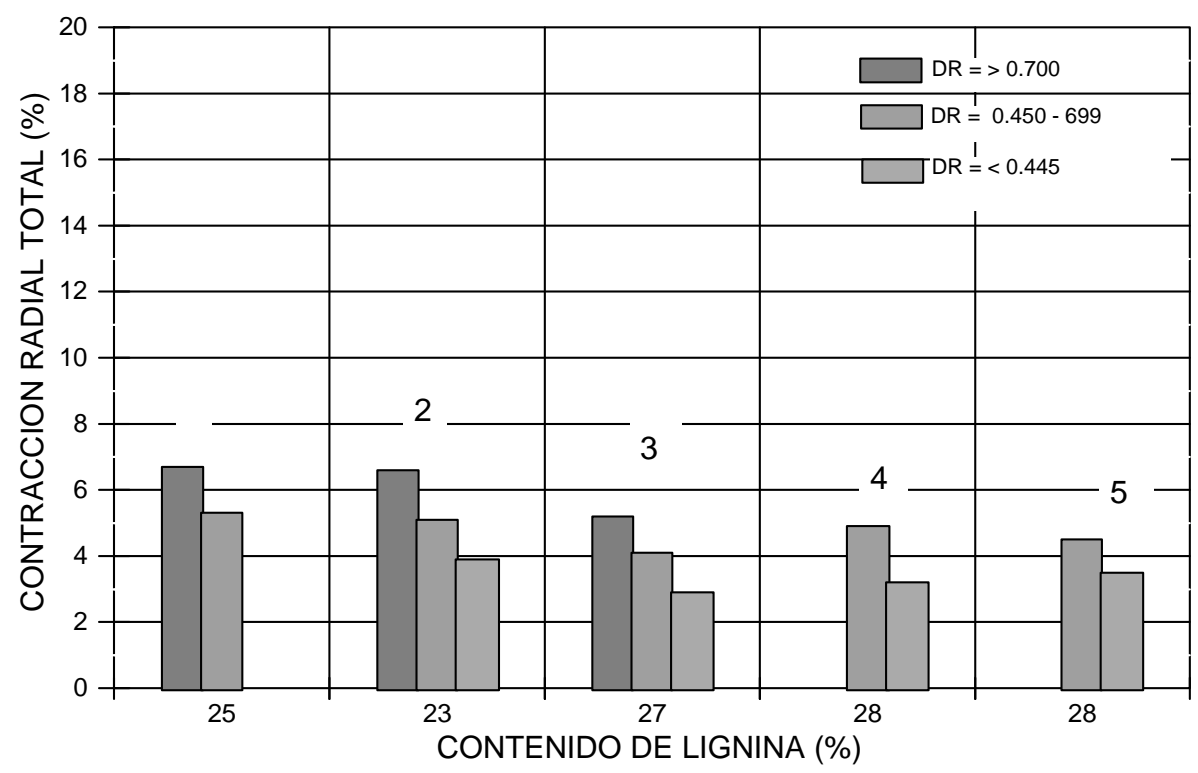

Figura 1. Valores promedio de contenido de lignina vs contracciones totales radial y tangencial de 26 maderas mexicanas 
Tabla 3 Resultados del análisis de regresión múltiple para contracción tangencial

\begin{tabular}{||c|c|c||}
\hline $\begin{array}{c}\text { CONTENIDO DE } \\
\text { LIGNINA (CL) }\end{array}$ & $\begin{array}{c}\text { DENSIDAD BASICA } \\
(\mathrm{DR})\end{array}$ & $\begin{array}{c}\text { ORDENADA AL } \\
\text { ORIGEN (b) }\end{array}$ \\
\hline 0.002 & 5.611 & 1.368 \\
\hline $\begin{array}{c}\text { ERROR ESTANDAR } \\
\mathrm{CL}\end{array}$ & $\begin{array}{c}\text { ERROR ESTANDAR } \\
\text { DR }\end{array}$ & $\begin{array}{c}\text { ERROR ESTANDAR } \\
\mathrm{b}\end{array}$ \\
\hline 0.033 & 0.987 & 1.156 \\
\hline \multicolumn{3}{|c|}{$\mathrm{R}^{2}=0.57$} \\
\hline
\end{tabular}

Tabla 4. Resultados del análisis de regresión múltiple para contracción radial

\begin{tabular}{||c|c|c||}
\hline $\begin{array}{c}\text { CONTENIDO DE } \\
\text { LIGNINA (CL) }\end{array}$ & $\begin{array}{c}\text { DENSIDAD } \\
\text { BASICA (DR) }\end{array}$ & $\begin{array}{c}\text { ORDENADA AL } \\
\text { ORIGEN (b) }\end{array}$ \\
\hline $\begin{array}{c}\text { ERROR ESTANDAR } \\
\text { CL }\end{array}$ & $\begin{array}{c}\text { ERROR } \\
\text { ESTANDAR } \\
\text { DR }\end{array}$ & $\begin{array}{c}9.670 \\
\text { ERROR ESTANDAR } \\
b\end{array}$ \\
\hline 0.096 & 2.872 & 3.363 \\
\hline \multicolumn{3}{|c}{$\mathrm{R}^{2}=0.46$} \\
\hline
\end{tabular}

En los dos casos analizados, se observa que ambas variables consideradas (densidad básica y contenido de lignina) tienen influencia, siendo la densidad básica la más importante, según lo indica el valor del coeficiente de determinación $\left(R^{2}\right)$. El valor de este coeficiente se incrementa sustancialmente al incorporar en la regresión el contenido de lignina, para el caso de la contracción tangencial; para el caso de la contracción radial, la incorporación del contenido de lignina no produce una modificación significativa al valor de $\mathrm{R}^{2}$ determinado con la densidad básica solamente (Fig. 2).

Los valores de los coeficientes de determinación obtenidos en los análisis de regresión simple entre contenido de lignina y contracciones son relativamente bajos, particularmente los de la contracción radial. Sin embargo, las tendencias son bastante definidas: a mayor contenido de lignina, menor valor de contracciones. Una posible razón que explicaría la evidente dispersión en los valores del contenido de lignina observada en la figura 3 es que los datos de contracciones y de contenido de lignina fueron obtenidos de fuentes diferentes. Particularmente, según opinión de los autores, los estudios de Acosta (1964) que fueron efectuados con una metodología relativamente antigua y de Pettersen (1984), realizados con algunas especies provenientes de Centroamérica y otras de México, los cuales es preciso corroborar. La inclusión de esta información en este trabajo se justifica por ser de los pocos estudios relativos a los componentes químicos de la pared celular efectuados con maderas mexicanas. 


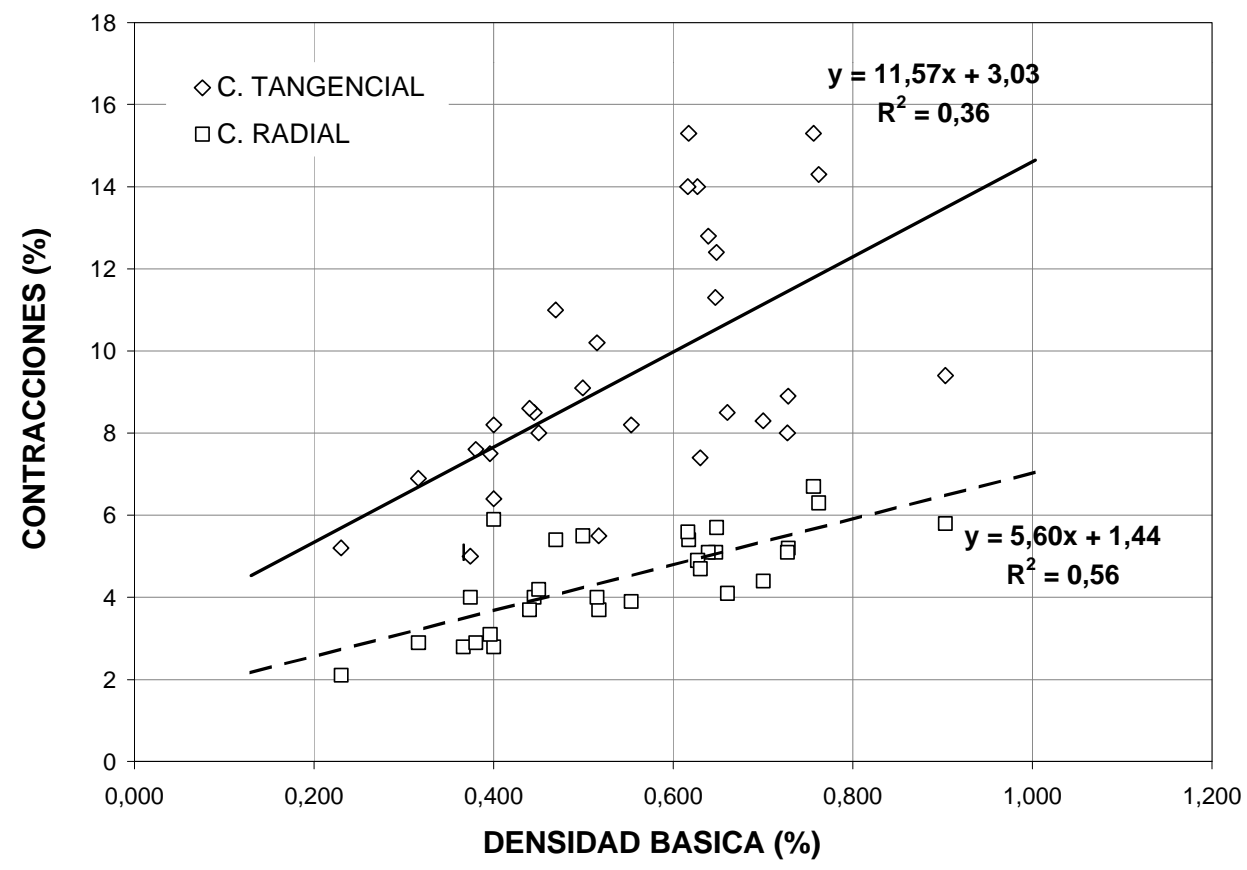

Figura 2. Relación densidad básica o relativa vs contracciones totales radial y tangencial de 26 maderas mexicanas

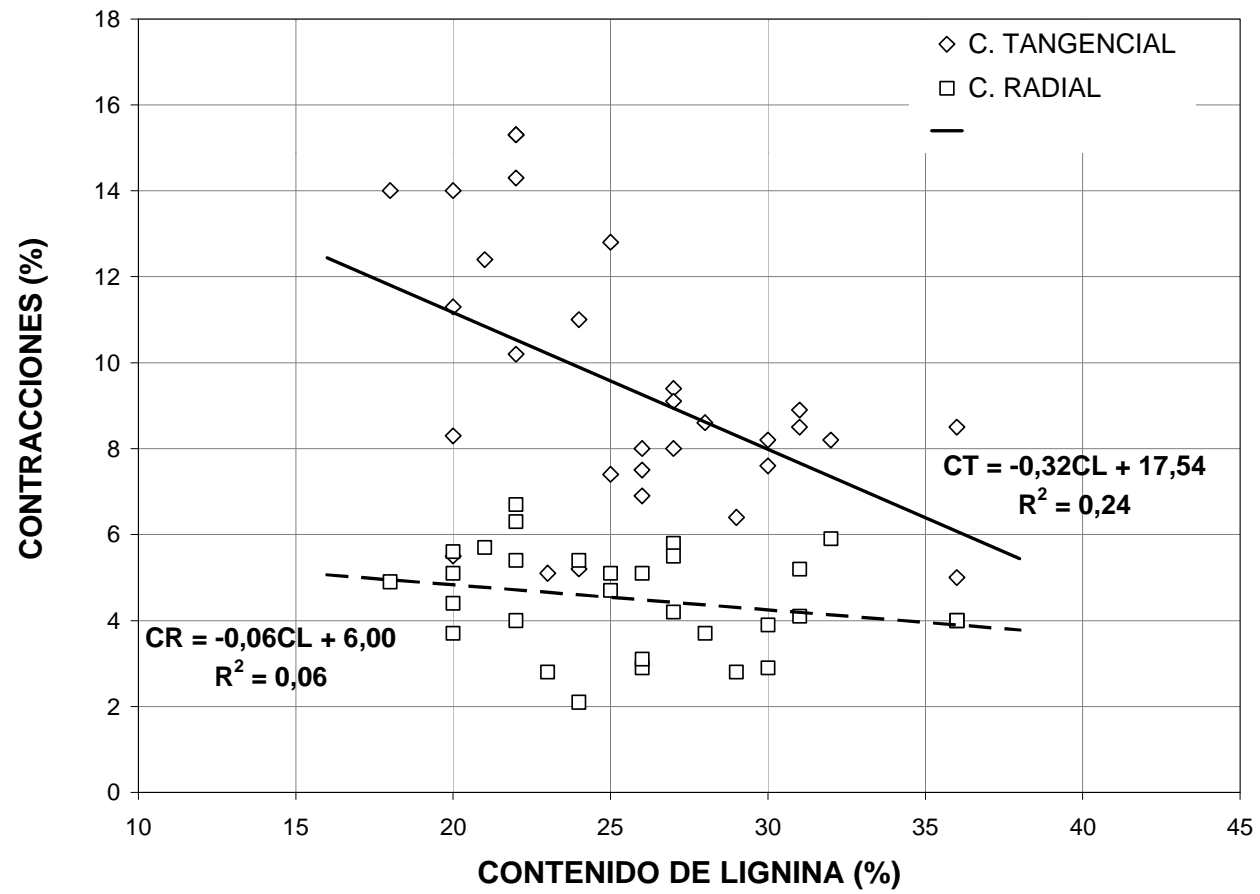

Figura 3. Relación contenido de lignina vs contracciones totales radial y tangencial de 26 maderas mexicanas 
Desde luego que el contenido de lignina no es la única variable que determina los cambios dimensionales de la madera. En la figura 1 se puede observar claramente que la densidad es un factor de primera importancia. Esto es consistente dentro de cada grupo. Las especies más densas presentan mayores valores de contracción, como consecuencia lógica de su mayor masa presente por unidad de volumen.

Resulta por demás interesante el comportamiento de las especies de angiospermas mexicanas de clima templado; sus valores de contracción radial son casi idénticos a los de las especies similares norteamericanas. Sin embargo, sus valores de contracción tangencial son mucho más altos, lo que debe estar explicado por otra fuente de variación distinta al contenido de lignina. Se presume que el origen de esta fuerte discrepancia en comportamiento es atribuible a la diferencia tan grande que existe entre el tamaño de los radios de las especies de los dos grupos, los que son mucho mayores en las especies mexicanas.

Los volúmenes de radios para especies mexicanas son de $37 \%$ y $27 \%$ para especies de densidad alta e intermedia, respectivamente, y de $32 \%$ y $16 \%$ para las categorías correspondientes de densidad, para las especies norteamericanas (Bárcenas y Dávalos, 1999a). El hecho de contar con un mayor volumen de radios, hace que las contracciones radiales sean menores y, por consiguiente, el valor del coeficiente de estabilidad T/R para las maderas mexicanas presenta valores mayores, es decir, son mucho más inestables que sus contrapartes norteamericanas. Esto tiene como consecuencia que su secado sea mucho más difícil, como bien se sabe. Los gruesos radios no se contraen fácilmente en su propia dirección longitudinal (la radial del árbol), ejerciendo una acción restrictiva al movimiento de la madera circundante y, sí pueden contraerse fácilmente en la dirección perpendicular (la tangencial del tronco), de ahí que la contracción tangencial de las maderas de angiospermas templadas mexicanas sea mayor. Este hecho subraya el concepto de que los radios son otro de los factores primordiales en el comportamiento de la madera (Bárcenas y Dávalos, 1999a; 1999b).

Otro factor de importancia que no se discute aquí, sino que únicamente se señala para completar los factores primordiales, es la cantidad de extractivos presentes en la madera. Estos están presentes en mucho mayor medida en las especies tropicales y, entonces, su influencia en las contracciones de éstas es mucho más marcada. Este punto se discute con mayor detalle en otras referencias recientes (Bárcenas y Dávalos, 1999a y 1999b).

\section{CONCLUSIONES Y RECOMENDACIONES}

El contenido de lignina desempeña un papel determinante en el comportamiento de la madera ante los cambios dimensionales por variaciones en el contenido de humedad, según se demuestra en los análisis estadísticos aquí presentados. Conjuntamente con la densidad relativa, el contenido de extractivos y el volumen de radios en la madera, parecería explicar el mayor porcentaje de contracción. La densidad básica es el parámetro más importante de los mencionados. Sin embargo, al eliminar esta variable del análisis (haciendo comparaciones entre grupos de especies con densidades similares), las diferencias encontradas obedecen a las diferencias en contenido de lignina entre grupos de especies. Las angiospermas de clima templado son las que presentan mayores contracciones y también los menores contenidos de lignina. Las especies más estables dimensionalmente son las coníferas, y después las maderas de angiospermas tropicales. Este patrón se repite tanto para especies originarias de México como de Estados Unidos, exceptuando, para este país, las especies tropicales, desde luego inexistentes.

Sería muy conveniente diseñar algún tipo de experimento con especies representativas de los varios grupos de especies para poder determinar con un mayor grado de precisión cuál es la real influencia de los diferentes factores que 
afectan el comportamiento de la madera ante cambios en su contenido de humedad. Este tipo de conocimientos permitiría un aprovechamiento más eficiente de nuestro recurso forestal, ya que permitiría la adopción de las secuelas de secado apropiadas para cada tipo de madera, si se conoce con precisión la cantidad de lignina presente, sus volúmenes de rayos y contenidos de extractivos; también indicaría cuáles especies requieren de mayor protección cuando se usen a la intemperie, al ser más susceptibles de deformarse. Es preciso, por tanto, contar con más información acerca de las cantidades en que se encuentran presentes las diversas sustancias químicas en la pared celular de las maderas mexicanas. Actualmente se cuenta con esta clase de información únicamente para un reducido número de especies nacionales.

\section{RECONOCIMIENTOS}

Los comentarios de los revisores anónimos fueron de suma importancia para la versión final de este documento. En particular, sus opiniones sobre el análisis estadístico y la discusión, influyeron sobremanera en la forma de presentar la información y de redactar el documento. Este trabajo se efectuó con el apoyo de los recursos fiscales asignados al Departamento de Productos Forestales y Conservación de Bosques del Instituto de Ecología, A.C. con la clave 902-13.

\section{REFERENCIAS}

Acosta C., M.R. 1964. Análisis químico y estudio de algunas de las características anatómicas de 23 especies de madera. Tesis. Facultad de Ciencias Químicas. Universidad Veracruzana. Instituto Mexicano de Investigaciones Tecnológicas, A.C. 96p.

Bárcenas P., G., R. Romero R. y R. Echenique M. 1980. Especies maderables de la Selva Lacandona. La Madera y su Uso en la Construcción No. 6. LACITEMA - INIREB. Xalapa, Ver. México. p:10.
Bárcenas P., G. 1995. Caracterización tecnológica de veinte especies maderables de la Selva Lacandona. Madera y Bosques 1(1):9-38.

Bárcenas P., G. s/f. Caracterización tecnológica de la madera de Liquidambar styraciflua. Informe de circulación restringida. Depto. Prod. Forestales y Conservación de Bosques. Instituto de Ecología, A.C.

Bárcenas P., G. y Dávalos S., R. 1999a. Shrinkage behavior of Mexican Woods. Journal of Tropical Forest Products 4(2). 36 p. (En prensa)

Bárcenas P., G. y Dávalos S., R. 1999b. Shrinkage values for Mexican Woods. Journal of Tropical Forest Products 5(1). 18 p. (En prensa)

Bland, D.E. 1971. The relation of lignin and polyphenol content of Tasmanian alpine ash (Eucalyptus delegatensis R.T. Bak) to shrinkage and recovery. Wood Science and Technology 5:1726.

Bosshard, H.H. 1956. Über die Anisotropie der Holzschwindung. Holz-als-Roh und Werkstoff 14:285-295.

Boyd, J.D. 1974. Anisotropic shrinkage of wood: Identification of the dominant determinants. Mokuzai Gakkaishi 20:473-482.

Candelario R., M.M. 1980. Estudio de la variación de algunas características físicas y mecánicas de la madera de Pinus ayacahuite var. veitchii y Pinus patula. Tesis. Departamento de Bosques. Universidad Autónoma de Chapingo. México.

Castañeda H., R. 1986. Composición química de la madera de tres especies tropicales de Jalisco. Tesis Licenciatura. Facultad de Agricultura. Universidad de Guadalajara.

Chafe, S.C. 1987. Collapse, volumetric shrinkage, specific gravity and extractives in Eucalyptus and other species. Part 2: The influence of wood 
extractives. Wood Science and Technology 21:27-41.

Davalos S., R.1994. Ayudas de diseño. In: Manual de construcción de estructuras ligeras de madera. Comisión Forestal de América del Norte. México, D.F., Madison, WI y Ottawa, Canadá.

De la Paz P.O., C., Dávalos S., R. y Quintanar I., A. 1995. Características de la madera de 20 especies de encino. Ponencia en el XII Congreso Mexicano de Botánica. Cuernavaca, Mor. México.

De la Paz P.O., C., Dávalos S., R. y Bárcenas P., G. 1999. Características de la madera de 2 especies de encino del estado de Puebla, México. Madera y Bosques. (En prensa)

Delgado F., E. 1980. Estudio analítico de los carbohidratos de cuatro especies de encino. Tesis Licenciatura. Fac. Ciencias Químicas. Universidad de Guadalajara.

Dickinson, F.E., Hess, R. W. y Wangaard, F.F. 1949. Properties and uses of tropical woods. Tropical Woods No. 95, June 1, 1949, p:1-145.

Echenique M., R. y V. Díaz G. 1969. Algunas características tecnológicas de la madera de once especies mexicanas. Boletín Técnico No. 27. Secretaría de Agricultura y Recursos Hidraúlicos. Subsecretaría Forestal y de la Fauna. Inst. de Investigaciones Forestales.

Echenique M., R. 1970. Veinticinco maderas tropicales mexicanas. Serie Maderas de México No. 1. Cámara Nacional de la Industria de la Construcción. México.

Fergus, B.J.; A.R. Procter; J.A.N. Scott y D.A.I. Goring. 1969. The distribution of lignin in sprucewood as determined by ultraviolet microscopy. Wood Science and Technology 3:117-138.

Fergus, B.J. y Goring, D.A.I. 1970. The location of guaiacyl and syringyl lignins in birch xylem tissue. Holzforschung 24(4):118-124.

Fuentes M., J.G. 1980. Estudio analítico de los carbohidratos de la madera de Quercus resinosa. Tesis Licenciatura. Escuela Politécnica. Universidad de Guadalajara.

Fuentes L., M. 1990. Propiedades físicomecánicas de cinco especies de encino Quercus spp del estado de Puebla. Tesis. Universidad Autónoma de Chapingo. México.

Forest Products Laboratory. 1989. Wood Handbook. Wood as engineering material. United States Departament of Agriculture. Forest Products Laboratory. Madison, WI.

Hernández-Pérez, J. 1995. Posibles usos de 5 encinos de Puebla de acuerdo a su composición química. In: Marroquín de la Fuente, J.S., ed. Proc. III Seminario Nacional sobre Utilización de Encinos, Nov. 4-6, 1992, Univ. Aut. de Nuevo León, Linares, N.L., México.

Higgins, N.C. 1957. The equilibrium moisture content-relative humidity of selected native and foreign woods. Forest Products Journal 7(10):371-377.

Islas O., E. 1992. Evaluación de cinco especies de pino para la producción de pulpas termomecánicas blanqueadas. Tesis de Maestría. Coordinación General de Investigación y Posgrado Ciencias Exactas e Ingeniería. Universidad de Guadalajara.

Kato, H. y Nakato, K. 1968. The transverse anisotropic shrinkage of wood and its relation to cell wall structure I. The lignin distribution in the radial and tangential walls of coniferous wood tracheids. Kyoto University Forestry Bulletin 40:284-292.

Kelsey, K.E. 1963. A critical review of the relationship between shrinkage and structure of wood. CSIRO Division of Forest Products. Technol. Paper No. 28:1-35. 
Montes R., E. 1991. Propiedades biológicas y físico-tecnológicas de las maderas de Pinus leiophylla y Pinus douglasiana. Tesis de Doctorado. Escuela de Graduados. Universidad de Guadalajara.

Nearn, W.T. 1955. Effect of water-soluble extractives on the volumetric shrinkage and the equlibrium moisture content of eleven tropical and domestic woods. Penn. State Univ., College of Agr. Bull. 598, University Park, Pa.

Novelo G., G. 1964. Posibles aplicaciones de la madera de cuatro especies tropicales con base en sus propiedades físicas y mecánicas. Tesis. Escuela Nacional de Agricultura. Chapingo, Edo. de México.

Ordóñez C., V.R., G. Bárcenas P. y A. Quiroz S. 1990. Características físicomecánicas de la madera de diez especies de San Pablo Macuiltianguis, Oax. Boletín Técnico La Madera y su uso No. 21. Instituto de Ecología, A.C. Univ. Aut. Metropolitana-Azcapotzalco. México.

Panshin, A.J. y de Zeeuw, C. 1970. Textbook of Wood Technology. Third Edition. Vol. 1, McGraw-Hill Book Co., Nueva York.

Panshin, A.J. y de Zeeuw, C. 1980. Textbook of Wood Technology. Fourth Edition. Vol. 1, McGraw-Hill Book Co., Nueva York.

Pettersen, R.C. 1984. The chemical composition of wood. In: Rowell, R.M., ed. The chemistry of solid wood. Advances in chemistry Series 207. Washington, D.C. American Chemical Society; 1984. Cap. 2.

Porres, J. y J. Valladares. 1979. Producción de pulpa y papel con materias primas autóctonas Centroamericanas I.
Producción de pulpa con 17 maderas tropicales del Petén, Guatemala, empleando el proceso Kraft o al sulfato. ICAITI.

Quirk, J.T. 1984. Shrinkage and related properties of Douglas-fir cell walls. Wood and Fiber 16:115-133.

SARH. 1982. Estudio promocional de 43 especies forestales tropicales mexicanas. Programa de Cooperación Científica y Técnica MéxicoYugoslavia. Publicación Especial. Secretaría de Agricultura y Recursos Hidráulicos. Subsecretaría Forestal y de la Fauna.

Schroeder, H.A. 1972. Shrinking and swelling differences between hardwoods and softwoods. Wood and Fiber 4(1):20-25.

Skaar, C. 1988. Wood-water relationship. Chapter 4. Springer Verlag Inc. New York. p:122-176.

Vidrio M., E. 1995. Estudio de las características físicas y químicas del tepeguaje (Lysiloma acapulcensis) y su potencial como materia prima en la obtención de pulpa celulósica. Tesis Licenciatura. División de Ingenierías. Centro Universitario de Ciencias Exactas e Ingeniería. Universidad de Guadalajara.

Villalvazo N., J. y Faix, O. 1981. Caracterización analítica de las ligninas de cuatro especies del género Quercus y sus posibilidades de aprovechamiento técnico. IMCyP Publicación Núm. 4, Nov. 1981. p:1-7.

Wiedenbeck, J.K., Hofmann, K., Peralta, P. Skaar, C. y P. Koch. 1990. Air permeability, shrinkage and moisture sorption of lodgepole pine stemwood. Wood and Fiber Science 22(3):229245. 